\title{
Analysis of the Fracture of Steel Reinforcing Bars under Low Cycle Fatigue
}

\author{
Lenin R. Abatta ${ }^{1}$, Carlos R. Arroyo ${ }^{2 *}$, Andrea V. Vaca ${ }^{2}$, Alexis Debut ${ }^{2}$, Leonardo Goyos ${ }^{1}$ and Reinaldo Delgado $^{1}$ \\ ${ }^{1}$ Departamento de Ciencias de la Energía y Mecánica, Universidad de las Fuerzas Armadas ESPE, P.O. Box 171-5-231B, Sangolqui, Ecuador
}

${ }^{2}$ Centro de Nanociencia y Nanotecnología, Universidad de las Fuerzas Armadas ESPE, P.O. Box 171-5-231, Sangolqui, Ecuador

*Corresponding author: Carlos R. Arroyo, Centro de Nanociencia y Nanotecnologia, Universidad de las Fuerzas Armadas ESPE, P.O. Box 171-5-231B, Sangolqui, Ecuador, Tel: +593-23989400 Ext: 2113; E-mail: ccarroyo@espe.edu.ec

Received date: March 16, 2016; Accepted date: April 06, 2016; Published date: April 11, 2016

Copyright: $\odot 2016$ Abatta LR, et al. This is an open-access article distributed under the terms of the Creative Commons Attribution License, which permits unrestricted use, distribution and reproduction in any medium, provided the original author and source are credited.

\begin{abstract}
In this work we have analyzed the dynamic behavior of steel reinforcing bars under low cycle force-controlled fatigue measurements. We compare the results obtained for rebars produced by three steel companies of Ecuador, which fulfill the technical requirements of the national standards specified in ASTM A706. The measurements were carried out by using an universal testing machine model MTS 810 . To determine the critical factors on the dynamic response of reinforcing bars, we have characterized the fractured section by macroscopic parameters and employing the Scanning Electron Microscopy (SEM). The results obtained point out that, although the three companies produce rebars with similar static behavior exist important differences on their dynamical properties.
\end{abstract}

Keywords: Low cycle fatigue; Steel reinforcing bars; ASTM A706

\section{Introduction}

Steel reinforcing bars, also known as rebars, are largely used in the construction industry to strengthen and hold concrete structures together. The mechanical parameters used for the characterization of rebars are the yield strength, the ultimate strength and the elongation. Such parameters fully characterize the static mechanical behavior of rebars while the dynamical characteristics are largely ignored in most of the specifications $[1,2]$. One of the reasons to not take into account the dynamical properties is the complexity and cost of the experimental setup. Another reason is the fact that the dynamic values critically depend on the manufacturing procedures and the alloy composition of the steel, which are hardly reproducible. However, the knowledge of the dynamic properties is essential for predicting the effects due to tsunamis, earthquakes, tornadoes and hurricanes on buildings and infrastructures. For example, during a seismic event, the concrete structures are subjected to lateral loads, which are mostly concentrated in the junctions (Figure 1) and results in structural failure $[3,4]$. Reversible loads applied to a structure produce the fracture of the concrete section (Figure 2) which evidence that the energy dissipation limit has been reached. In the current study, the dynamic response of steel reinforcing bars has been evaluated under low cycle forcecontrolled fatigue measurements. We compare the results obtained for rebars of $12 \mathrm{~mm}$ of diameter produced by three different Ecuadorian companies, which have achieved the technical standards for commercialization.

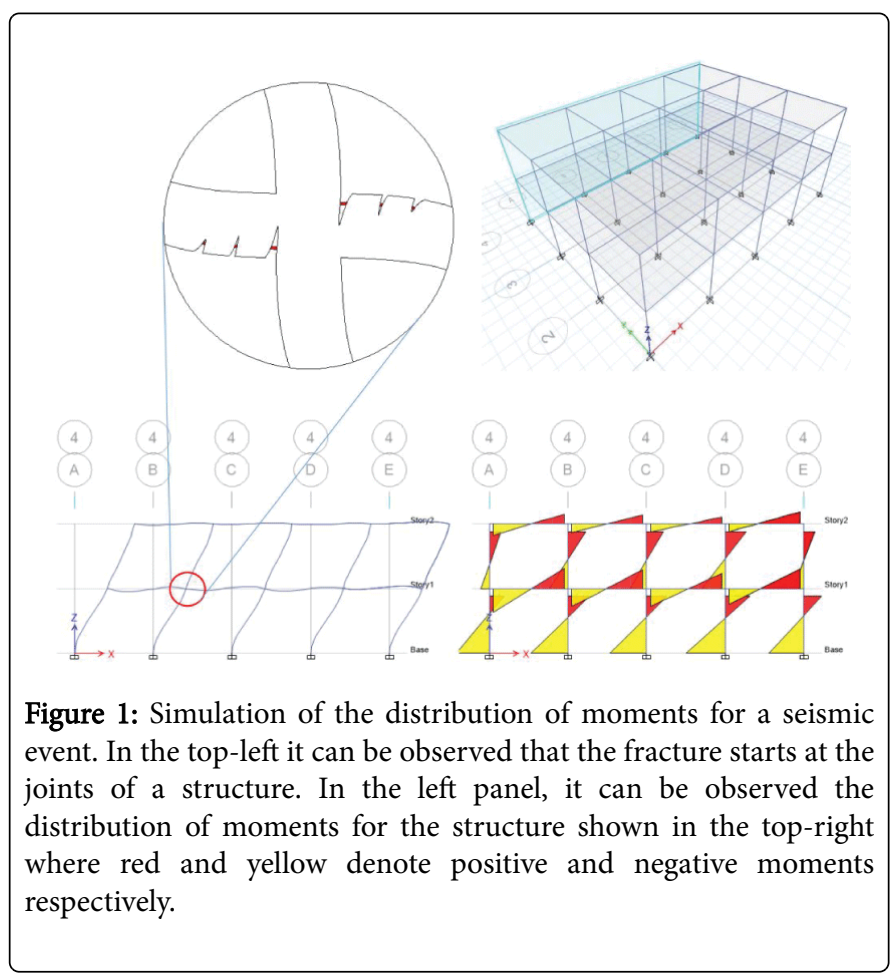

\section{Materials and Methods}

This research has been focused on corrugated steel rebars of $12 \mathrm{~mm}$ of diameter and yield strength of $412 \mathrm{MPa}$ conforming to ASTM A706. The rebars are currently made and sold in Ecuador by three important companies, which will be denoted as yellow, white and red in the rest of the paper. The rebars, directly obtained from the market, were cut in lengths of $40 \mathrm{~cm}$ and marked every $60 \mathrm{~mm}$ in order to measure the percent of elongation at the end of each test. 


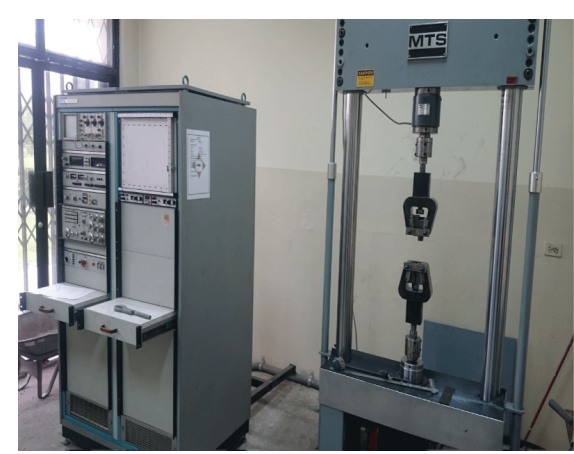

Figure 2: Universal testing machine MTS 810 utilized for low cycle force-controlled fatigue measurements.

Then, each rebar was anchored to the universal testing machine MTS 810 by its ends using grips and a longitudinal force was applied. To simulate the loading and unloading tension of a seismic event, we have applied a sinusoidal wave at a frequency $1 \mathrm{~Hz}$. To ensure reproducibility, we have performed low cycle force-controlled fatigue measurements in 10 different rebars of each company.

\section{Results and Discussion}

In Figure 3, it is represented a typical low cycle force-controlled fatigue test performed at room temperature $25^{\circ} \mathrm{C}$. The low cycle forcecontrolled fatigue tests are performed under tensile stress and focused in the plastic deformation stage once the elastic limit of the material has been reached. The rebar is elongated during $1 \mathrm{~s}$ and then the force is relaxed. Successive cycles were applied until the breaking of the rebar. At the end of the test the number of cycles before the breaking and the percentage of elongation of the fractured segment are registered for each rebar.

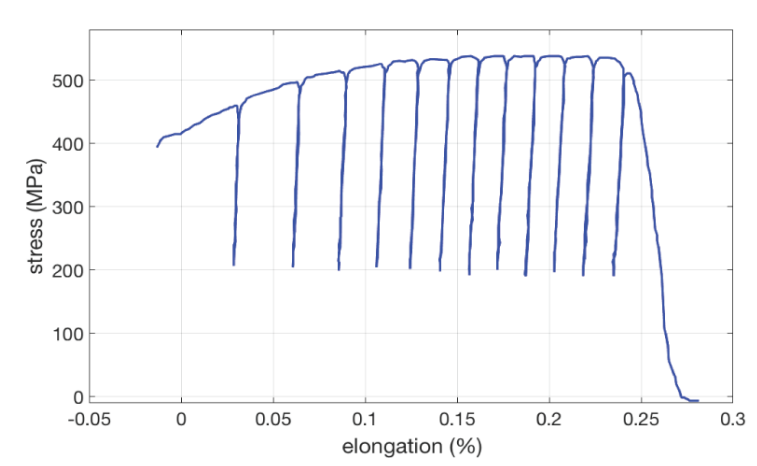

Figure 3: Representative low cycle force-controlled fatigue measurement performed on a steel reinforcing bar. In this particular example the number of cycles before the fracture was 12 and the final elongation $0.25 \%$.

The microstructures observed on the SEM images of the fractured sections, shown in Figure 4, are typical of a fracture due to fatigue [5]. It is important to note that in all the experiments performed the cracking or fracture starts at the end or beginning of a corrugation.
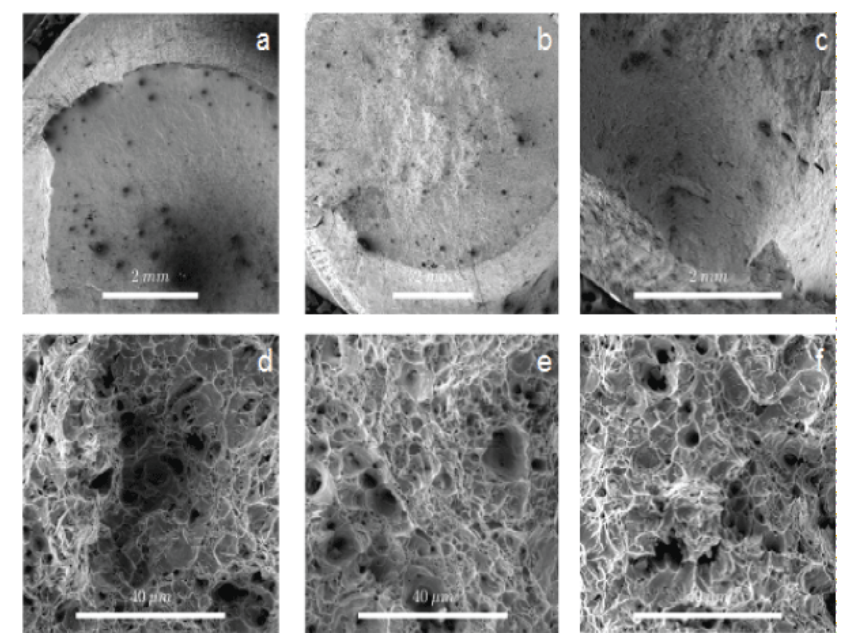

Figure 4: SEM micrographs of the fractured sections. (a-c) Low magnification images (32X) obtained from samples yellow, white and red respectively. (d-f) High magnification images (3000X) obtained from samples yellow, white and red respectively.

As observed in Figure 5a, all three type of samples (e.g. white, yellow and red) have similar percentage of elongation, which is according to the Ecuadorian standards, as it is mentioned in INEN 2167 (2011). The Yellow rebar has better behavior under dynamic loads resulting in up to 32 cycles, as observed in Figure $5 b$.

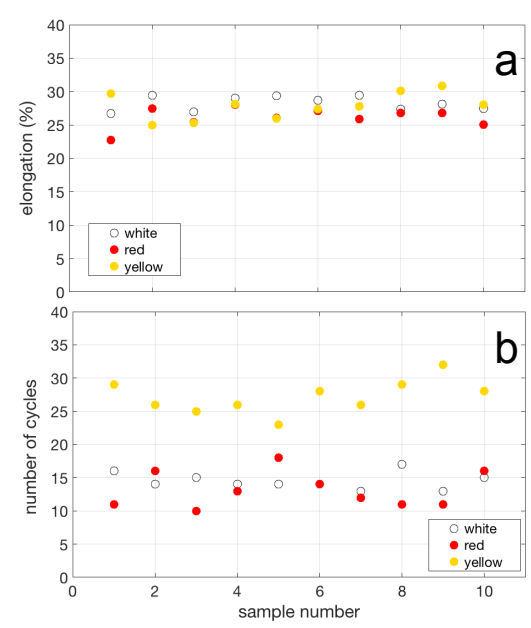

Figure 5: Results obtained from the low cycle force-controlled fatigue measurements of white, red and yellow rebars. (a) Elongation as a function of the number of tested samples. (b) Number of cycles before the fracture of the rebar as a function of the number of tested samples.

\section{Conclusions}

With the aim of characterize the dynamic behavior of steel reinforcing bars, we have performed low cycle force-controlled fatigue test on rebars of three different Ecuadorian steel companies. Although 
Citation: Abatta LR, Arroyo CR, Vaca AV, Debut A, Goyos L, et al. (2016) Analysis of the Fracture of Steel Reinforcing Bars under Low Cycle Fatigue. Biol Med (Aligarh) 8: 285. doi:10.4172/0974-8369.1000285

Page 3 of 3

the static characteristics of the rebars are quite similar, there are notable differences on the dynamic response. As it is observed in the results of our measurements, the number of cycles before the breaking is always higher for Yellow rebars, resulting in a better dynamic behavior than the others. However, the unknown about the alloy composition and the procedures of production make it hard to conclude which are the critical factors. Further work is in progress in order to determine the essential parameters, which determine the dynamic behavior of steel reinforcing bars.

\section{References}

1. Malvar LJ (1998) Review of static and dynamic properties of steel reinforcing bars. ACI Materials Journal 95: 609-616.
2. Schijve J (2001) Fatigue of structures and materials. Springer, Netherlands.

3. Billah AM and Alam MS (2012) Seismic performance of concrete columns reinforced with hybrid shape memory alloy (SMA) and fiber reinforced polymer (FRP) bars. Construction and Building Materials 28: 730-742.

4. Ou YC, Tsai MS, Chang KC and Lee GC (2010) Cyclic behavior of precast segmental concrete bridge columns with high performance or conventional steel reinforcing bars as energy dissipation bars. Earthquake Engineering \& Structural Dynamics 39: 1181-1198.

5. Soltani A (2011) Bond and serviceability characterization of concrete reinforced with high strength steel. University of Pittsburgh. 